\title{
Pandemic COVID-19 caused by SARS-CoV-2: genetic structure, vaccination, and therapeutic approaches
}

\author{
Hany E. Marei ${ }^{1}$ (D) Asmaa Althani ${ }^{2} \cdot$ Nahla Afifi $^{3} \cdot$ Giacomo Pozzoli $^{4} \cdot$ Thomas Caceci $^{5} \cdot$ Franco Angelini $^{6}$. \\ Carlo Cenciarelli ${ }^{7}$
}

Received: 2 April 2021 / Accepted: 5 August 2021 / Published online: 16 August 2021

(c) The Author(s), under exclusive licence to Springer Nature B.V. 2021

\begin{abstract}
We give a summary of SARS-genetic CoV-2's structure and evolution, as well as current attempts to develop efficient vaccine and treatment methods for SARS-CoV-2 infection, in this article. Most therapeutic strategies are based on repurposing of existing therapeutic agents used against various virus infections and focused mainly on inhibition of the virus replication cycle, enhancement of innate immunity, and alleviation of CRS caused by COVID-19. Currently, more than 100 clinical trials on COVID-19 aim to provide robust evidence on the efficacy of the currently available anti-SARS-CoV-2 antiviral substances, such as the nucleotide analogue remdesivir, the antimalarial drug chloroquine, and drugs directed against docking of SARS-CoV-2 to the membrane-associated angiotensin-converting enzyme 2 (ACE2) such as transmembrane protease serine 2 (TMPRSS2). The current vaccination campaign is ongoing worldwide using different types of vaccines such as Pfizer-BioNTech and Moderna, Johnson \& Johnson, Oxford-AstraZeneca, Novavax, and others with efficacy ranging from 72-95\%. In March 2021 Germany limited the use of the Oxford-AstraZeneca COVID-19 vaccine to people 60 years of age and older due to concerns that it may be causing blood clots. Further study and more data are needed to confirm the safety of different available vaccines.
\end{abstract}

Keywords COVID-19 genomics · Vaccine $\cdot$ Antiviral agents $\cdot$ Therapeutic approach

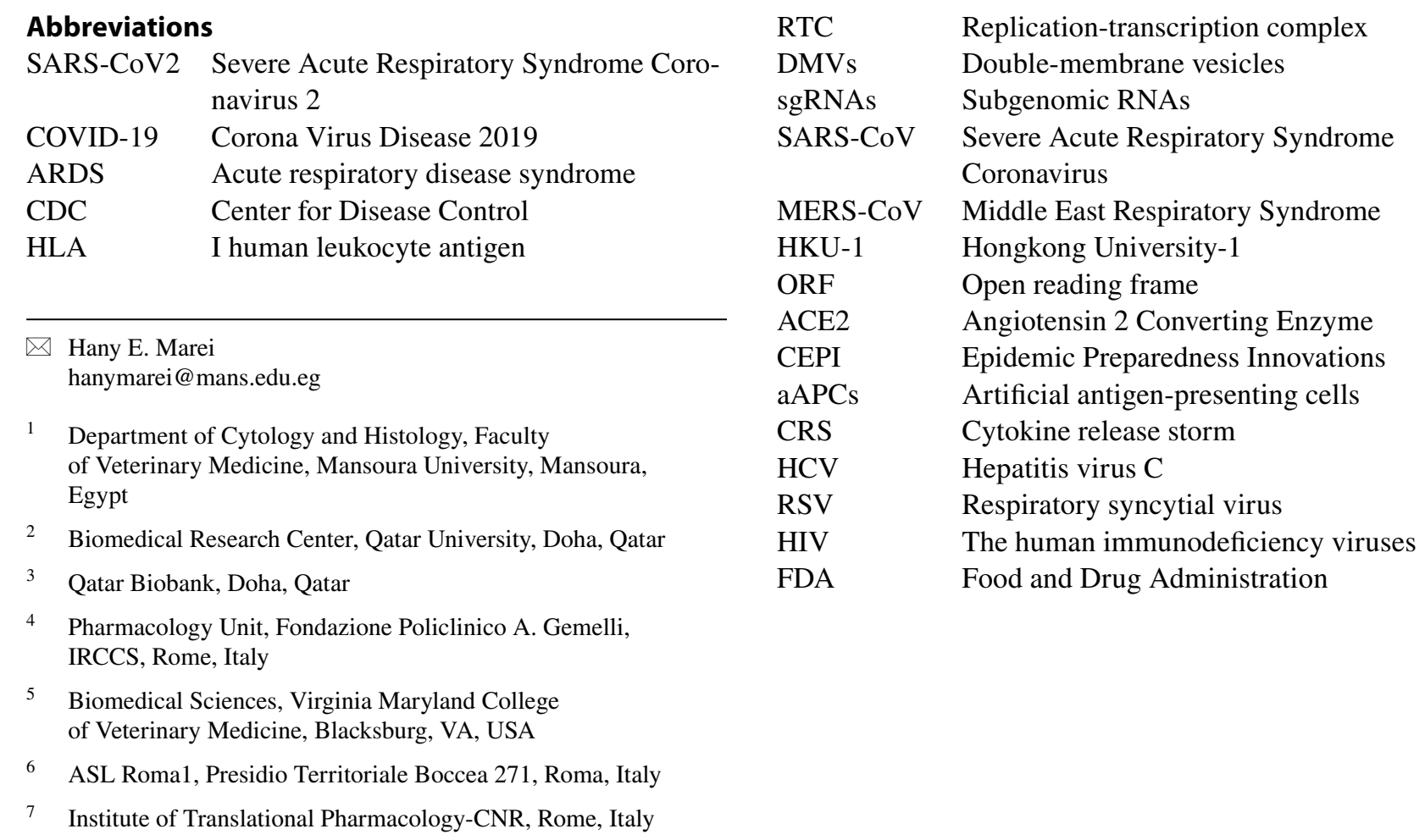




\section{Introduction}

At the end of 2019, an alarming infectious disease caused by a newly discovered coronavirus was first recorded in the Chinese city of Wuhan (Hubei province, [1]). The rapid, disturbing world- wide transmission of this virus, named COVID-19, resulted in declaration of a global health emergency by the World Health Organization on 30 January 2020 [2]. As of April 1, 2021, there have been $129 \mathrm{M}$ global confirmed cases, $2.82 \mathrm{M}$ recovered cases of COVID-19, and $2.82 \mathrm{M}$ deaths reported to WHO. Mutti et al. [3] reported that $\sim 18 \%$ of COVID-19 patients develop interstitial pneumonia, and $\sim 5 \%$ of them develop acute respiratory disease syndrome (ARDS), which is mostly fatal [3]. The risk of ARDS rises with age, and almost all deaths are seen in patients with pre-existing chronic conditions [19, 20].

Increasing evidence has shown sustained human-tohuman transmission, resulting in many cases exported across the globe by travelers (CDC, 2020). A role for specific class I human leukocyte antigen (HLA) alleles in shaping the anti-viral immune response to COVID-19 has been suggested, and permissive roles of HLA-C*01 and $\mathrm{B} * 44$ towards SARS-CoV-2 infection have also been postulated [4]. This information may help to identify sub-populations at risk, facilitating the prioritization of vaccination programs.

In this review we provide a brief introduction of the epidemiology of SARS-CoV-2 and describe its theoretical potential evolution, based on what is known about preexisting similar coronaviruses. We provide an update on the potential origin and evolution of the mutated genetic structure of SARS-CoV-2, and of the current efforts to provide effective vaccination and therapeutic strategies.

\section{Genome structure of SARS-CoV-2 compared to other coronaviruses}

The production of an efficient vaccine/treatment necessitates a thorough understanding of the genetic, genomic and proteomic architecture of Severe Acute Respiratory Syndrome Coronavirus 2 (SARS-CoV2). According to the phylogenetic tree of coronaviruses (CoVs), CoVs belong to the order Nidovirales, which includes the family Coronaviridae and the subfamily Coronavirinae. Four genera are included in the subfamily Coronavirinae: Alphacoronavirus, Betacoronavirus, Gammacoronavirus, and Deltacoronavirus [5].

The CoVs genome structure is $\sim 30 \mathrm{~kb}$ single-stranded positive-sense RNA (+ ssRNA) with 3'-poly-A tail, and 5 '-cap structure. The CoV's genome is translated into two long polypeptides ( $\mathrm{Pp} 1 \mathrm{a}$ and $\mathrm{pp} 1 \mathrm{~b}$ ) that are processed into 16 nonstructural proteins (nsps) forming the replication-transcription complex (RTC) of double-membrane vesicles (DMVs, [6]). Adopting a discontinuous transcription mechanism, the RTC synthesizes a nested set of subgenomic RNAs (sgRNAs) that possess common 5'-leader and 3'-terminal sequences [7]. Five ORFs constitute the genome of CoV: The first ORF (ORF1a/b) encodes 16 nonspecific proteins (nsp1-16). Others encode at least four main structural elements: spike (S), membrane (M), envelope (E), and nucleocapsid $(\mathrm{N})$ proteins $[7,8]$. The $\mathrm{S}$ proteins form the spikes on the viral surface that mediate attachment of virions to host receptors [9-16].

The structural proteins include the structural Spike (S), Envelope (E), Membrane (M), Nucleocapsid (N), Hemagglutinin esterase (HE) and Helicase $(\mathrm{H})$ proteins. The nonstructural proteins (nsp) include Proteases, papain-like proteases (PLP or PLpro) and 3C-like protease (3CLpro), and Replicase proteins [17]. The spike (S) protein protrudes on the viral surface, and play a crucial role in the fusion of the viral particles to specific receptors on host cells, while the envelope (E) protein is known to have viroporin activity which is essential for completion of the infection cycle. The nucleocapsid $(\mathrm{N})$ protein merge with the viral single (+) stranded RNA genome forming a nucleoprotein complex which is maintained within the capsid comprised in part of the matrix $(\mathrm{M})$ protein. The protease proteins have been a target of anti-viral action by lopinavir/ritonavir, with and without arbidol, an anti-envelope viral indole derivative [18].

\section{Evolution and novel genetic structure of severe acute respiratory syndrome coronavirus 2 (SARS-CoV2)}

By the end of January 2020, the genomic sequence of SARS$\mathrm{CoV}-2$ was determined and the information released [19]. To understand the origin of this novel coronavirus and how it is related to existing coronavirus members, the genomic sequences of SARS-CoV-2 were compared to the severe acute respiratory syndrome (SARS) caused by a SARSassociated coronavirus that was first identified at the end of February 2003, and Middle East respiratory syndrome or (MERS-CoV) that was first identified in Saudi Arabia in 2012 [20]. Interestingly, the sequence of SARS-CoV-2 shared a higher homology with SARS-CoV than that of MERS-CoV. Moreover, poor sequence homology was noted mainly in the ORF1ab and spike (S-protein) gene. Bat-CoV and SARS-CoV-2 showed $92 \%$ sequence similarity. MERS$\mathrm{CoV}$ and SARS-CoV-2 nucleocapsid analysis indicated only $65 \%$ identity. Molecular characterization of nucleocapsids from various coronaviruses revealed that SARS-CoV 2 is more related to SARS-CoV 1 and bat-CoV. SARS-CoV 2 exhibited less resemblance with MERS-CoV. SARS-CoV 2 
showed less similarity to MERS-CoV. Thus, either SARSCoV-1 or bat-CoV may be the source of SARS-CoV-2 evolution [21].

MERS-CoV was found to share genetic homology with many bat $\mathrm{CoV}$ species worldwide, which included the Egyptian tomb bats, African bats [22, 23], Italian bats [24], and Chinese bats [25]. Similar to SARS-CoV, MERS-CoV also has two domains $\mathrm{S} 1$ and $\mathrm{S} 2$, of which $\mathrm{S} 1$ is the variant peptide that binds to the receptor dipeptidyl peptidase 4 (DPP4). $\mathrm{S} 1$ domain is located in the 240 residue of the C-terminal receptor binding domain and are composed of a core and external subdomain unit enabling their attachment, fusion, and entry into the host cells [26].

Recently evolved SARS-CoV-2 (COVID-19), the seventh known coronavirus has shown similar properties of SARS$\mathrm{CoV}$ with the presence of a highly variable S1 sub-unit of spike protein. It was also found that about five of the six residues of S1 domain differ in SARS-CoV-2 compared to the S1 domain of SARS-CoV virus [27]. Further, the presence of polybasic cleavage site namely the three O-linked glycan in the intersection of the S1 and S2 sub-domains also appear to play a vital role in the infective properties of the virus [28]. Thus, it is inferred that the variation in the $\mathrm{S} 1$ and S2 domains of the spike protein act as the major site of mutation, insertion, and deletion of genes during every transformation of the SARS-CoV-2 virus. This enables the evolution of the new infective virus forms that are capable of efficiently binding to the receptor binding domain, further enabling the effective invasion of viral particles into the human cells.

Whether or not the transmission pathway from bats to humans may include other intermediate hosts is still a matter of speculation. Based on genomic profiling of the two mostly related human- infecting SARS-CoV, it is now evident that the genetic distance of SARS-CoV-2 is much closer to the human-infecting SARS-CoV than is the MERS-CoV [29]. Although the origin of the virus remains unresolved, Wacharapluesadee et al. extended the geographic distribution of genetically diverse SARS-CoV from Japan and China to Thailand over a 4800-km range. Cross-border surveillance is urgently needed to find the immediate progenitor virus of SARS-CoV-2 [30].

In a recent report, WHO-convened global study of origins of SARS-CoV-2. The study aimed at identifying the zoonotic source of SARS-CoV-2, the route of introduction to the human population, and potential intermediate hosts. This is crucial to reduce further risks of the emergence of new virus zoonotic disease. The study concluded that at this stage, it is not possible to determine precisely how humans in China were initially infected with SARS-CoV-2. However, all available evidence suggests that SARS-CoV-2 has a natural animal origin and is not a manipulated or constructed virus. SARS-CoV virus most probably has its ecological reservoir in bats [31].

Coronaviruses are known to utilize S-protein to facilitate entry into the host cells by binding to host receptors. The two functional units of the S-proteins (S1 and S2) play crucial roles in host receptor binding. Individually, the C-terminal RBD is directly attached to surface receptors [32]. The S-protein has been reported to have the most variable sequence of amino acid residues [33]. Despite the observation that the SARS-CoV-2 S-protein has a low overall homology to SARS-CoV, the RBD domain of SARSCoV-2 does show several sequences with close homology to that of SARS-CoV_Tor2 and HP03-GZ01. The receptor complex of SARS-CoV S-protein has five amino acids residues that are crucial for human-to-human and cross-species transmission [34]. In the SARS-CoV-2 S-protein, Tyr491 is the only conserved residue; the other 4 residues are not preserved. Similar to SARS-CoV, SARS-CoV-2 also uses the angiotensin-converting enzyme 2 (ACE2) for cell entry [35].

To prove the ability, and the nature, of the interaction of SARS-CoV-2 with human ACE2 molecules, Xu et al. [36] have studied the structural model of the SARS-CoV-2 S-protein using a computer-guided homology model based on the crystal structure of SARS coronavirus S-protein (PDB accession: 6ACD) as a template [37].

\section{Repurposing existing antiviral therapy for COVID-19}

Until now, no specific antiviral therapy has been available to treat COVID-19 respiratory damage. Nevertheless, efforts are ongoing to test several antiviral drugs both at the preclinical and clinical levels. Based on their mode of action, antiCOVID-19 therapies might target either enzyme involved in viral replication; or interfere with the viral entry into the host cells. Others might target the host immune system to enhance host immunity; or inhibit the cytokine release storm (CRS) associated with the massive damage of lung tissues (Fig. 1).

Based on previous therapeutic experience with SARS and MERS, several options might be available: these include small-molecule antiviral drugs, monoclonal antibodies, interferon therapies, oligonucleotide-based therapies, peptides, and vaccines [38].

Based on the genomic code of SARS-CoV-2, a set of structural and non-structural proteins have been identified as potential therapeutic targets. Replicating enzymes (3-chymotrypsin-like protease, papain-like protease, helicase, and RNA-dependent RNA polymerase) that are essential in the viral life cycles as well as $\mathrm{S}$ protein, which is known to play a decisive role in SARS-CoV-2 entry into the host cells via ACE2 receptor [39], may be options. Drugs directed against 
Fig. 1 Various therapeutic targets for SARS-Co-v-2
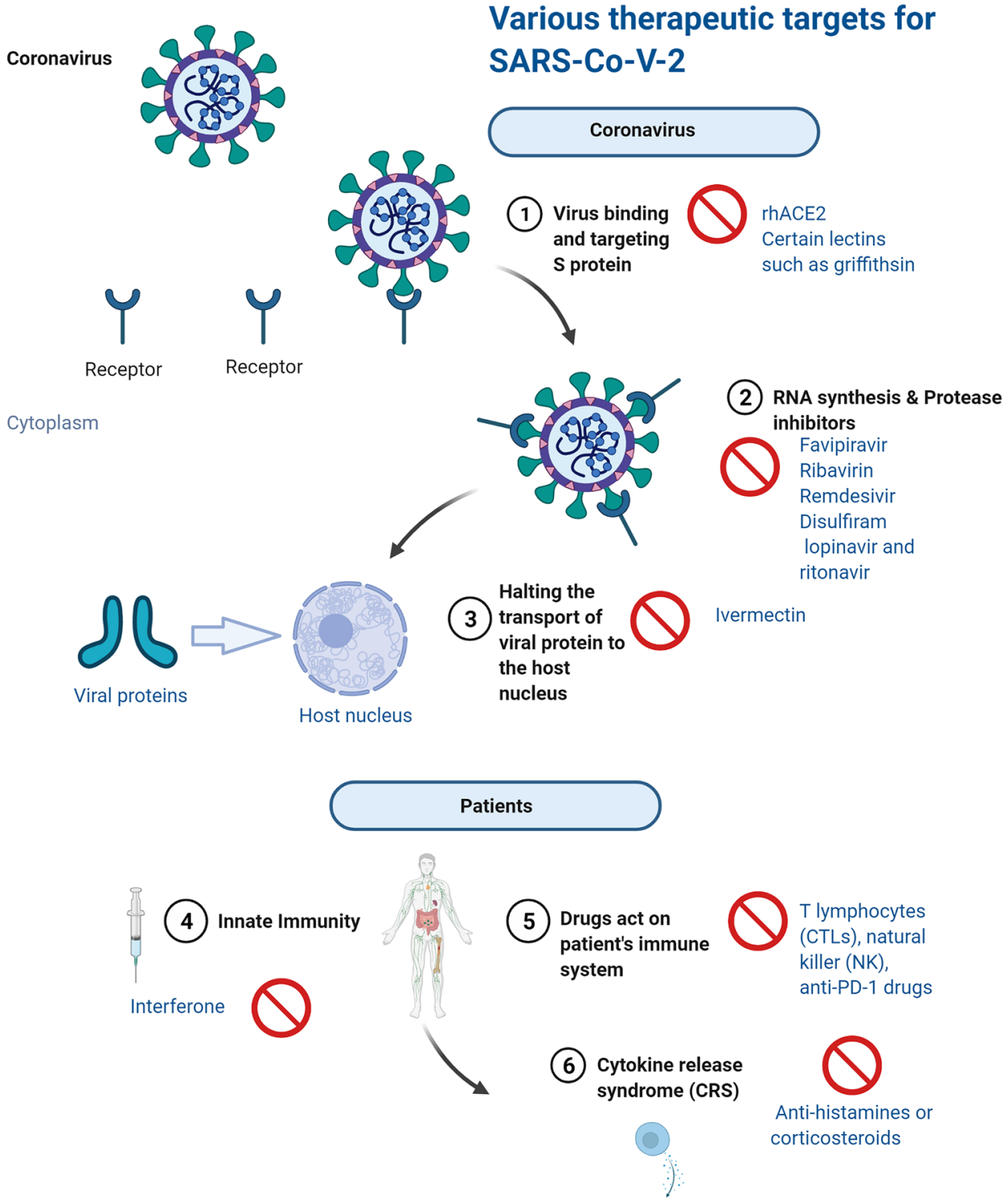

This figure shows the various therapeutic targets of SARS-CoV-2

and drugs used against them.

docking of SARS-CoV-2 to the membrane-associated angiotensin converting enzyme 2 (ACE2) such as transmembrane protease serine 2 (TMPRSS2) are also under current evaluation [40].

When comparing them to SARS and MERS enzymes, the catalytic sites of the SARS-CoV-2 replication enzymes and the key drug-binding pockets in viral enzymes seem to be highly conserved. Therefore, repurposing existing MERS and SARS antiviral agents such as nucleoside analogues (favipiravir and ribavirin) and/or experimental nucleoside analogues (remdesivir and galidesivir) may be a promising therapeutic approach (Fig. 1). The nucleoside analogues act against a wide range of RNA viruses (including coronaviruses) principally by targeting the RNA-dependent polymerase, thus inhibiting viral RNA synthesis [41].
The antiviral inhibitory effect of favipiravir (T-705) has been confirmed against several RNA viruses such as influenza, Ebola, yellow fever, chikungunya, norovirus and enterovirus. Favipiravir has been approved to treat influenza [41]. Using Vero cells, favipiravir has been demonstrated to be effective against SARS-CoV-2 (EC50 $=61.88 \mu \mathrm{M}$ in Vero E6 cells). It effectively inhibits the RNA-dependent polymerase of SARS-CoV-2 by structurally resembling endogenous guanine [34, 42]. Currently, the efficacy of favipiravir plus interferon- $\alpha$ (ChiCTR2000029600) and favipiravir plus baloxavir marboxil (ChiCTR2000029544) are being evaluated in COVID-19 patients.

Ribavirin is approved for hepatitis virus $\mathrm{C}(\mathrm{HCV})$ and respiratory syncytial virus (RSV). The efficacy of ribavirin against SARS and MERS has also been evaluated, but it has 
been reported to induce severe anaemia when administrated in a high dose [39]. Whether or not ribavirin can confer efficient potency against SARS-CoV-2is has yet to be clarified.

Remdesivir (GS-5734) is a prodrug whose structure resembles adenosine. As an adenine derivative, it was initially developed to treat Ebola [43]. Previous studies have proven that remdesivir induces productive antiviral activity against SARS-CoV and MERS-CoV, in vitro and in vivo $[44,45]$. A recent study showed that remdesivir inhibited SARS-CoV-2 $($ EC50 $=0.77 \mu \mathrm{M}$ in Vero E6 cells, $[42,46])$. A COVID-19 patient has recovered after receiving intravenous remdesivir [47].

Galidesivir (BCX4430) as an adenosine analogue has been evaluated against HCV and yellow fever, and has been proven to provide antiviral effects against SARS and MERS [39].

Protease inhibitors are used against several virus infections and disease conditions such as SARS, MERS, HIV, and alcohol dependence. The rationale behind the use of protease inhibitors (such as disulfiram, lopinavir and ritonavir) is their ability to inhibit papain-like protease. The protease proteins have been a target of anti-viral action by lopinavir/ritonavir, with and without a rbidol, an anti-envelope viral indole derivative [18]. Inhibition of MERS and SARS papain-like protease has been demonstrated in cell cultures, but clinical evidence still needs to be developed. Currently both lopinavir and ritonavir, previously tested as HIV protease inhibitors, are under clinical trial in COVID19 patients. In a non-randomized open label trial, lopinavir and ritonavir administration were associated with improved clinical outcome in SARS patients, and have been hypothesized to induce such favorable outcomes via inhibition of 3-chymotrypsin-like protease [39]. Whether HIV protease inhibitors can improve the clinical outcome in COVID-19 patients is still in need of clarification.

Targeting the $\mathrm{S}$ protein of SARS-CoV-2 is another tactic to prevent viral entry into the host cells. Certain lectins (such as griffithsin, which is derived from red algae) have been reported as able to bind to oligosaccharides associated with the HIV glycoprotein 120 and SARS-CoV S glycoprotein [39]. In a phase I clinical trial griffithsin has been evaluated against HIV using different routes of administration as gels or in enemas. Whether the same approach could be implemented for SARS-CoV-2 is still in need of study.

The FDA-approved anti-parasitic ivermectin has previously shown antiviral effects against several RNA viruses such as HIV and dengue virus [48]. Ivermectin acts by dissociation of the IMP $\alpha / \beta 1$ heterodimer, thereby halting the transport of viral protein cargo to host cell nuclei. This results in inhibition not only of the viral replication cycle but also the endogenous antiviral defense mechanisms in the host cells. In a recent in vitro study, ivermectin was able to reduce the viral RNA load up to 5000 -fold $48 \mathrm{~h}$ after infection with SARS-CoV-2 [49]. The next logical step after establishing the safety profile and efficacy against SARSCoV-2 would be the initiation of clinical testing to estimate the appropriate dose necessary to inhibit the SARS-CoV-2 viral replication cycle.

Inhibition of viral entry into host cells by blocking virus-cell membrane fusion seems to be another promising approach against SARS-CoV-2 infections. Molecules designed to occupy the ACE2 receptors in competition with SARS-CoV-2 might inhibit the ability of SARS-CoV-2 to enter the host cells. Soluble recombinant human Angiotensin-converting Enzyme 2 (rhACE2) blocks the interaction between the S protein of SARS-CoV-2 and ACE2, and inhibits SARS-CoV-2 replication in cellular and embryonic stem cell-derived organoids by a factor 1000-5000 times [50]. Administration of rhACE2 preserved the integrity of pulmonary vasculature and prevented acute respiratory distress syndrome (ARDS, [51]). Currently, the biological and physiological role of rhACE2 is being evaluated in a small pilot study in China (NCT04287686), and the safety and tolerability of APN01 (rhACE2 produced by Apeiron Biologics) are currently being assessed in a placebo-controlled, double blinded, dose-escalation clinical trial.

Glycosylation of ACE2 receptors has also been demonstrated to inhibit the replication of SARS-CoV [52].

The cytotoxic T lymphocytes (CTLs) and natural killer cells (NK) are known to have an essential role in fighting viral infection. In SARS-CoV-1 infection [53], and tumorigenesis [54] persistent immune activation is associated with lymphocyte exhaustion, showing significantly higher levels of exhaustion markers. For example programmed death-1 (PD-1), is higher compared to healthy controls [55]. The use of anti-PD-1 drugs to trigger exhausted $\mathrm{T}$ cells by blocking PD-1 in cases of viral infections has been suggested and the same concept might be applied to SARS-CoV-2 infection [56].

Recently, a solidarity clinical trial for COVID-19 treatments has been launched by the World Health Organization and partners to evaluate the effects of drugs used to treat COVID-19 on three important outcomes in COVID-19 patients: mortality, need for assisted ventilation and duration of hospital stay. The solidarity study concluded that all 4 treatments evaluated (remdesivir, hydroxychloroquine, lopinavir/ritonavir and interferon) had little or no effect on overall mortality, initiation of ventilation and duration of hospital stay in hospitalized patients (https://www.who.int/ emergencies/diseases/novel-coronavirus-2019/global-resea rch-on-novel-coronavirus-2019-ncov/solidarity-clinicaltrial-for-covid-19-treatments). Other studies have shown the inefficacy of Hydroxychloroquine in COVID-19 patients [57]. 


\section{Ongoing SARS-CoV-2 vaccination}

Elucidation of the genomic sequence of SARS-CoV-2, first published on 11 January 2020, was the first crucial step toward designing genome-based specific vaccines and therapeutic antibodies. The next step is to test the safety and efficacy of such novel vaccines in clinical trials. Although this represents the classical pathway of vaccine development, given the current pandemic nature of SARS-CoV-2, this long-term solution, while needed, is a problem. Much more rapid intervention is urgently required to halt a lethal pandemic.

Based on data released by The Coalition for Epidemic Preparedness Innovations (CEPI, a collaborative effort between global health authorities and vaccine developers to support the development of vaccines against COVID19) the vaccine $R \& D$ landscape now includes 115 vaccine candidates. These include approaches using nucleic acid (DNA and RNA), virus-like particles, peptides, viral vector recombinant protein (replicating and non-replicating), as well as live attenuated-virus and inactivated-virus (Fig. 2 and Table 1).

The DNA and mRNA vaccines have significant advantages due to the ease of antigen manipulation and potential for speedy production. Viral-vector-based vaccines provide a much stronger immune response, higher levels of protein expression, and therefore long stability. Adjuvant vaccines against COVID-19 are expected to enhance the immune response and decrease the vaccine dose. The crucial role played by the viral spike (S) protein in viral uptake via the ACE2 receptor has prompted the creation of vaccines targeting the $\mathrm{S}$ proteins. These are intended to trigger the production of neutralizing antibodies against it. That would prevent the SARS-CoV-2 uptake via the ACE2 receptor. Whether the different variants of the $S$ protein used in different vaccines under trial are related to each other or the genomic epidemiology of the disease is not yet clear.

Several B and T cells epitopes that map identically to SARS-CoV-2 proteins have been identified [58]. Immune targeting of these epitopes may potentially offer protection [58]. The time needed for traditional vaccine development would normally average 10 years, but given the lethal nature of the pandemic, unprecedented efforts have currently been employed to produce an effective vaccine by late 2020 and early 2021. The situation has necessitated adoption of a novel paradigm relevant not only in the development phase but also in scaling up manufacturing capacity. With respect to the concerted global efforts for speedy production of COVID-19 vaccine, it is crucial to assess efficacy through development of animal models. Furthermore, availability of Biosafety-level 3 facilities is crucial for performing animal studies involving live-virus challenges [59].
Demonstration of the crucial role played by the S protein in initiating of infections, together with the data generated by sequencing of the SARS-CoV-2 genome led many of the vaccine developers to assess the efficacy of multiple nucleic acid-based vaccine candidates based on the genomic sequence of S protein. The safety and efficacy of a synthetic mRNA (Moderna's mRNA-1273, NCT04283461) and synthetic DNA (Inovio Pharmaceuticals, INO-4800) encoding the $\mathrm{S}$ protein of SARS-CoV-2 are currently being tested.

Other lipid-nanoparticle-encapsulated S protein-based mRNA vaccines are also expected to trigger an effective antiviral immune response upon intramuscular injection. The immunogenicity of genetic vaccines depends to a great extent on the route of administration and the amount of delivered plasmid. In a Phase I/II an ongoing clinical trial, the genetic sequence of the $\mathrm{S}$ protein from the $\mathrm{nCoV}-19$ was introduced into a non-replicating adenovirus vector. The adenovirus tropism to both respiratory and gastrointestinal epithelium (the two sites for which SARS-CoV-2 has a predilection) and its ability to exploit the ACE- 2 receptor as its main entry route into the host cells potentially makes the non-replicating adenovirus an appropriate vector for a DNA-based vaccine. However, the potential for eliciting an immune response against the vector genes (and to a lesser extent against the transgenes) is still in need of clarification (NCT04324606).

Mimicking previous trials with influenza and Ebola viruses, a stabilized subunit vaccine based on molecular clamp technology is under trial for SARS-CoV-2. This approach might allow recombinant viral proteins to remain stable in pre-fusion form, allowing investigators to test their ability to induce the production of neutralizing antibodies [60].

By linking nanoparticles to SARS-CoV-2 S protein antigenic epitopes, a nanoparticle-based vaccine able to trigger an antigen-specific lymphocyte proliferation (as well as cytokine production) is expected to enter phase I trial this summer by Novavax, Inc [61]. The $\mathrm{S}$ protein epitopes are stably expressed in a baculovirus system [62].

Cytotoxic $\mathrm{T}$ cells and genetically modified artificial antigen-presenting cells (aAPCs) that are specific for SARSCoV-2 $\mathrm{S}$ antigenic epitopes could trigger the naïve $\mathrm{T}$ cells in the human body, leading to their differentiation and proliferation. Ongoing clinical trials are now assessing the safety and immunogenicity of aAPCs alone and in combination with antigen-specific cytotoxic T cells (NCT04299724, NCT04276896).

The strategies adopted in the development of vaccines are very different from each other and consequently, so are the types of vaccine that can protect against infection (Fig. 2). In particular, the researchers are working on different types of vaccines: RNA vaccine, DNA vaccine, protein vaccine, inactivated viral vaccine, viral vector vaccine, attenuated 


\section{Various types of Vaccine Candidates for COVID-19*}
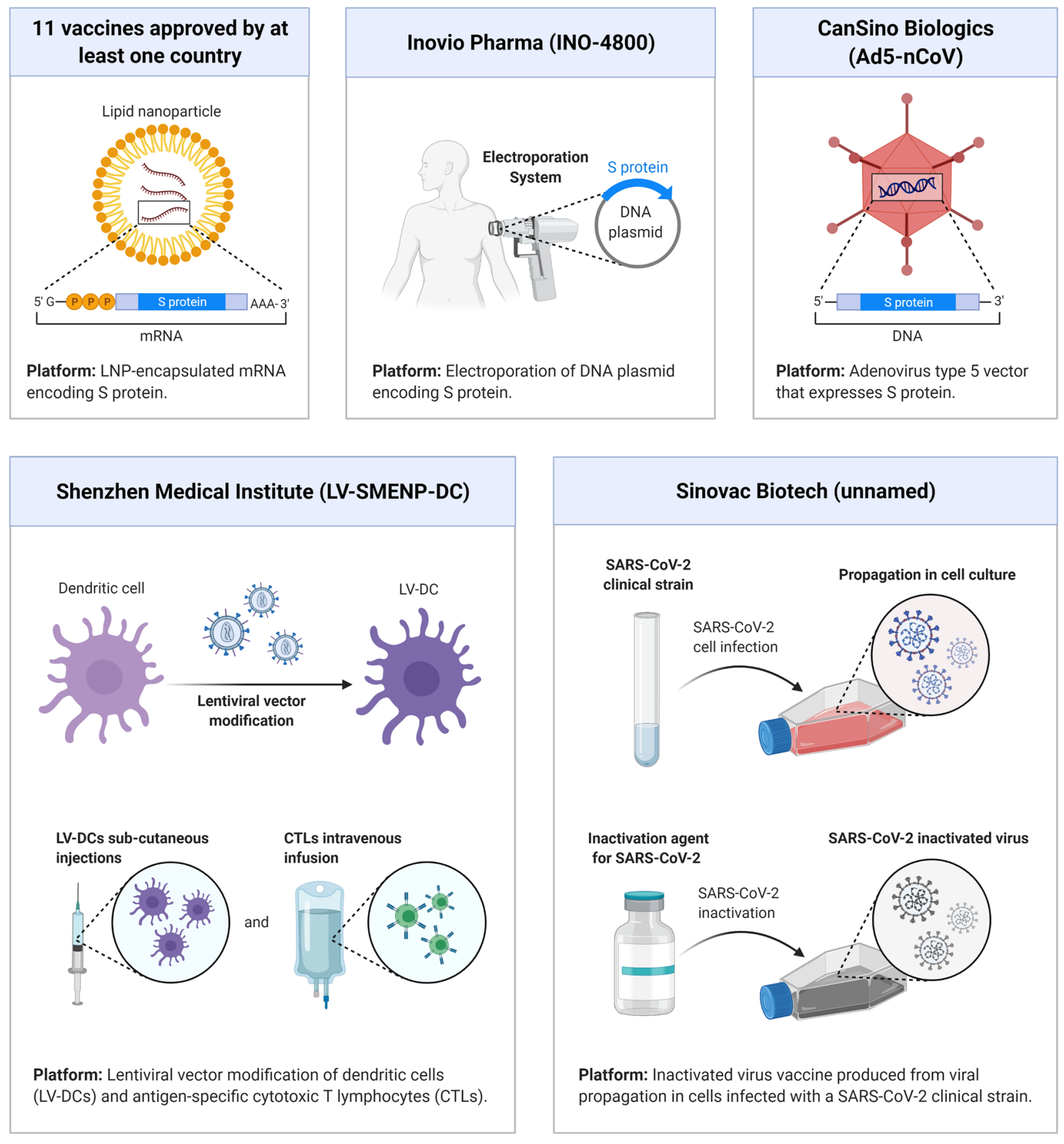

*As of April 2020

Fig. 2 Various types of vaccines candidate for COVID-19

live vaccine. However, the situation of vaccine development is rapidly and continuously evolving, as is the pandemic. Specifically, an overview of the clinical trial phases of the anti SARS-CoV2 vaccines is reported for the period of November/December 2020.
Worldwide, China currently presents two vaccines in phase 1/2 (SARS-CoV-2 Inactivated Vaccine, LV-SMENP DC) and two in phase 3 (Vero cell, Ad5-nCoV); the United States two in phase 3 (mRNA-1273 sponsor Moderna TX. Inc., AZD-1222 sponsor Astra Zeneca Iqvia Pty Ltd); India 
Table 1 Various types of vaccines

\begin{tabular}{|c|c|c|c|c|}
\hline No. & Name & Type & Approval & Trials/country \\
\hline \multicolumn{5}{|c|}{11 vaccines approved by at least one country } \\
\hline 1 & FBRI EpiVacCorona & Protein subunit & 1 country & 2 trials in 1 country \\
\hline 2 & Moderna mRNA-1273 & RNA & 38 countries & 5 trials in 1 country \\
\hline 3 & Pfizer/BioNTech BNT162b2 & RNA & 60 countries & 9 trials in 9 countries \\
\hline 4 & CanSinoAd5-nCoV & Non-Replicating Viral Vector & Approved in 3 countries & 6 trials in 6 countries \\
\hline 5 & GamaleyaSputnik V & Non-Replicating Viral Vector & Approved in 26 countries & 15 trials in 6 countries \\
\hline 6 & Oxford/AstraZenecaAZD1222 & Non-Replicating Viral Vector & Approved in 48 countries & 18 trials in 13 countries \\
\hline 7 & Serum Institute of IndiaCovishield & Non-Replicating Viral Vector & Approved in 11 countries & 2 trials in 1 country \\
\hline 8 & Bharat BiotechCovaxin & Inactivated & Approved in 1 country & 5 trials in 1 country \\
\hline 9 & Sinopharm (Beijing)BBIBP-CorV & Inactivated & Approved in 14 countries & 6 trials in 7 countries \\
\hline 10 & Sinopharm (Wuhan) & Inactivated & Approved in 2 countries & 6 trials in 7 countries \\
\hline 11 & SinovacCoronaVac & Inactivated & Approved in 7 countries & 11 trials in 5 countries \\
\hline \multicolumn{5}{|c|}{20 vaccines in phase 3 clinical trials } \\
\hline 12 & NovavaxNVX-CoV2373 & Protein subunit & & 5 trials in 6 countries \\
\hline 13 & Anhui Zhifei LongcomRBD-Dimer & Protein subunit & & 5 trials in 1 country \\
\hline 14 & MedicagoPlant-based VLP & VLP & & 3 trials in 2 countries \\
\hline 15 & InovioINO-4800 & DNA & & 6 trials in 3 countries \\
\hline 16 & AnGesAG0301-COVID19 & DNA & & 4 trials in 1 country \\
\hline 17 & ModernamRNA-1273 & RNA & Approved in 38 countries & 5 trials in 1 country \\
\hline 18 & CurevacCVnCoV & RNA & & 5 trials in 4 countries \\
\hline 19 & Pfizer/BioNTechBNT162b1 & RNA & & 5 trials in 3 countries \\
\hline 20 & Pfizer/BioNTechBNT162b2 & RNA & Approved in 60 countries & 9 trials in 9 countries \\
\hline 21 & GamaleyaSputnik V & Non-Replicating Viral Vector & Approved in 26 countries & 15 trials in 6 countries \\
\hline 22 & Janssen (Johnson \& Johnson)Ad26.COV2.S & Non-Replicating Viral Vector & & 6 trials in 17 countries \\
\hline 23 & CanSinoAd5-nCoV & Non-Replicating Viral Vector & Approved in 3 countries & 6 trials in 6 countries \\
\hline 24 & Oxford/AstraZenecaAZD1222 & Non-Replicating Viral Vector & Approved in 48 countries & 18 trials in 13 countries \\
\hline 25 & Serum Institute of IndiaCovishield & Non-Replicating Viral Vector & Approved in 11 countries & 2 trials in 1 country \\
\hline 26 & Chinese Academy of Medical SciencesInactivated & Inactivated & & 5 trials in 3 countries \\
\hline 27 & Kazakhstan RIBSPQazCovid-in & Inactivated & & 3 trials in 1 country \\
\hline 28 & Bharat BiotechCovaxin & Inactivated & Approved in 1 country & 5 trials in 1 country \\
\hline 29 & Sinopharm (Beijing)BBIBP-CorV & Inactivated & Approved in 14 countries & 6 trials in 7 countries \\
\hline 30 & Sinopharm (Wuhan)Inactivated & & Approved in 2 countries & 6 trials in 7 countries \\
\hline 31 & SinovacCoronaVac & Inactivated & Approved in 7 countries & 11 trials in 5 countries \\
\hline
\end{tabular}

By 19 February 2021, 11 COVID-19 vaccines approved by at least one country, 20 vaccines in phase 3 clinical trials, 39 vaccines in phase 2 clinical trials, and 27 vaccines in phase 1 clinical trials

with two phase 1/2 vaccines (BBV-152, Novel Corona Virus. 2019- nCov Vaccine); Brazil one in phase 3 (PROFISCOV); Korea a phase $1 / 2$ vaccine (INO.4800); Turkey one in phase 3 (CoronaVac); Russia one in phase 3 (Gam-COVID Vac). In Europe: United Kingdom one in phase 1/2 (LNP-nCoVsaRNA sponsor Imperial College (London) and one in phase 2/3 (ChAdOx1 nCoV-19 sponsor University Oxford); Belgium and Germany one in phase $1 / 2(\mathrm{CVnCoV}$ Vaccine sponsor CureVac AG); Germany one in phase 3 (BNT 162b2 sponsor BioNTech SE + Pfizer). In Italy, AIFA with a statement authorized on 31/07/2020, the testing of phase I on the anti-COVID-19 vaccine produced by Italian Biotechnological company ReiThera. This is a phase I study which aims to evaluate the safety and immunogenicity of the GRAd-COV2 vaccine. The vaccine development project is supported by the Ministry of Research (MUR) with the National Research Council (CNR) of Italy and by the Lazio Region. The trial will be conducted at the National Institute for Infectious Diseases "L. Spallanzani" in Rome and at the Clinical Research Center in Verona (Table 1). The results of the phase $2 / 3$ clinical trial have been published in a recent publication in "The Lancet" magazine. This referred to the ChAd vaccine $0 \times 1 \mathrm{nCoV}-19$ from the Biopharmaceutical company Astra Zeneca on a study carried out on 4 cohorts, and showed significant efficacy of $70.4 \%$ after 2 doses and a protection level of $64.1 \%$ after at least one standard dose, 
against symptomatic disease, without safety problems. Furthermore, according to the authors who followed the evolution of vaccines administration at various age groups of the population, they showed a greater tolerability for the vaccine in older adults than in younger adults. It also has similar immunogenicity in the three different age groups after the second dose. However, further studies are deemed necessary to evaluate the efficacy of the vaccine in individuals with comorbidities. On November 18, 2020, Pfizer published the preliminary results of the study in phase 3 (US National Library of Medicine ClinicalTrials.gov) announcing the conclusion that the trial has shown a 95\% efficacy of the vaccine against COVID-19, starting on day 28 after administration of the first dose. When participants were age-stratified, the efficacy observed in adults over the age of 65 years was greater than $94 \%$. Considering that the safety data required by the US Food and Drug Administration (FDA) for the authorization for emergency use (EUA) has been reached, the data were presented not only to these Authorities but also to other Agencies around the world, including AIFA in Italy and EMA in Europe. Regarding the Russian Sputnik V vaccine, preliminary data published on the website sputnikvaccine.com show an efficacy of over $95 \%$ at 42 days from the first dose. The preliminary results of the Phase 3 study by the Moderna company were also published, which reports that the $0.1773 \mathrm{mRNA}$ vaccine is $100 \%$ effective against severe COVID and was found to be safe and well tolerated with an efficacy rate of $94.5 \%$. (Fig. 2 and Table 1).

\section{Vaccines Authorized by FDA (Food and Drug Administration), EMA (European Medicines Agency) and AIFA (Italian Medicines Agency), from December 2020 to January 2021}

On 10 December 2020, the article "Safety and Efficacy of the BNT162b2 mRNA COVID-19" was published in The New England Journal of Medicine with the results of a Phase III study [54]. On 11 December 2020, the Food and Drug Administration (FDA) issued the authorization for emergency use and allowed the distribution of the PfizerBioNTech COVID -19 vaccine in the United States.

On 21 December 2020 the EMA (European Medicines Agency) issued a press release recommending conditional marketing authorization for the vaccine developed by BioNThec and Pfizer, to prevent COVID-19 in people 16 years of age and older. The scientific opinion of the EMA paved the way for the first authorization to place on the market a COVID-19 vaccine in the EU by the European Commission, with all the guarantees, controls and obligations that this implies. The AIFA (Italian Medicines Agency) also authorized the marketing of the Pfizer-BioNTech vaccine on $22 / 12 / 2020$ to be administered to subjects aged 16 or over and to be administered as a cycle of two injections.

On 18 December 2020, the FDA issued the authorization for emergency use (EUA) for the second vaccine for the prevention of COVID-19. The authorization allows the distribution of the Moderna COVID-19 vaccine in the United States for use in individuals aged 18 years or older. On 30 December 2020, The New England Journal of Medicine [55], analyzing data from approximately 30,000 participants enrolled in 100 clinical research centers, reported that the potential vaccine was found to be safe and well tolerated with an efficacy rate of $94.5 \%$.

On January 29, 2021, the EMA, after careful evaluation of the quality, safety and efficacy of the AstraZeneca vaccine and Oxford University ChAdOx1 nCOV19 (AZD1222) vaccine with a non-replicating viral vector, recommended authorization for release to the market. This vaccine stands at an average efficacy level of 70\%. In line with the decision of the EMA, on 30 of January 2021, the AIFA also authorized its use in Italy, underlining its preferential use in subjects between the ages of 18 and 55 for whom more solid evidence is available.

On 30 January-February 2021. AIFA recommended the AstraZeneca vaccine for over $55 \mathrm{~s}$. Waiting to acquire further data from the studies currently underway for the Astra Zeneca vaccine, it suggested preferential use in populations for which there is more solid evidence, namely subjects between 18 and 55 years old. It also reiterated that, on the basis of immunogenicity results and safety data, the benefit/risk ratio of this vaccine is favorable even in older subjects who do not have specific risk factors. In March 2021 Germany limited the use of the Oxford-AstraZeneca COVID-19 vaccine to people 60 years of age and older due to concerns that it may be causing blood clots [63].

\section{Vaccines without marketing authorization in Europe: SPUTNIK V-Gam-COVID-Vac of Gamaleya Res. Institute and Ministry of Health Russian Federation}

On February 2, 2021, The Lancet magazine Logunov et al. [64] published ad interim results of the phase 3 study on GamCOVID -Vac (SPUTNIK V) in which it is confirmed an efficacy against COVID 19 of $91.6 \%$ and good tolerance in a large cohort of patients. The randomized, double-blind, placebocontrolled study was carried out in 25 hospitals in Moscow and involved 21,977 adults randomly assigned to the vaccine group $(n=16,501)$ or the placebo group $(n=5476)$ with a $3: 1$ ratio. The vaccine comprises two vector components, rAd26$\mathrm{S}$ and rAd5-S carrying the full-length gene for the S. SARS COV 2 glycoprotein, administered i.m. with an interval of 21 days between the first rAd26 dose and the second rAd5. The European Medicines Agency (EMA) has not received to 
Table 2 Most common Antiviral drugs for COVID-19 and their mechanism of action

\begin{tabular}{lll}
\hline & Drugs & Target/mechanism of action \\
\hline 1 & Favipiravir & $\begin{array}{c}\text { Inhibits the RNA-dependent polymerase of SARS-CoV-2 } \\
\text { by structurally resembling endogenous guanine } \\
\text { Prodrug whose structure resembles adenosine }\end{array}$ \\
2 & Ribavirin & An inhibitor of the viral RNA-dependent, RNA polymerase \\
3 & Remdesivir (GS-5734) & Adenosine analogue \\
4 & Galidesivir (BCX4430) & Protease inhibitors \\
5 & Lopinavir/ritonavir & Targeting the S protein of SARS-CoV-2 \\
6 & Griffithsin & \\
\hline
\end{tabular}

date a cyclic review or marketing authorization application for SputnikV (Gam-COVID-Vac) vaccine developed by the Gamaleya National Center for Epidemiology and Microbiology in Russia.

\section{The problem of variants: the English variant (Kent)}

What is worrisome about variants is not where they come from, but the mutations they contain. Virus B.1.1.7 is characterized by a deletion in the spike protein and a mutation in N501Y that increases its transmissibility, as well as a potentially important mutation in the furin cleavage site. These mutations are set against the background of an unusually high number of other mutations that make B.1.1.7 distinct. In the case of B.1.351, the key mutation that makes it a threat to the effectiveness of a vaccine is the E484K mutation in the spike protein, also seen in P.1. The English variant was followed by the "South African" variant B.1.351 which contains a mutation reported to reduce the effectiveness of the ChAdIx Astra Zeneca vaccine, therefore South Africa is removing the vaccine from its vaccination schedule.

To summarize the current situation of COVID-19 vaccination, the following millstones and important dates are denoted. By 11 January 2020, the genetic sequence of SARS-CoV-2 was identified. By June 2020, dozens of vaccine candidates have prepared to prepare for global vaccination programs to immunize against COVID-19 infection [65]. On 24 June 2020, China approved the CanSino vaccine for limited use in the military, and two inactivated virus vaccines for emergency use in high-risk occupations. On 11 August 2020, Russia announced the approval of its Sputnik V vaccine for emergency use. The Pfizer-BioNTech partnership submitted an EUA request to the FDA for the mRNA vaccine BNT162b2 on 20 November 2020. As of 21 December, many countries and the European Union had authorized or approved the Pfizer-BioNTech COVID19 vaccine. On 11 December 2020, the United States Food and Drug Administration (FDA) granted an Emergency Use Authorization (EUA) for the Pfizer-BioNTech
COVID-19 vaccine [66]. A week later, they granted an EUA for mRNA-1273, the Moderna vaccine. On March 31, 2021, the Russian government announced that they had registered the first COVID-19 vaccine for animals named Carnivac-Cov, it is an inactivated vaccine for carnivorous animals, including pets. In June 2021, a report revealed that the UB-612 vaccine, developed by the USbased COVAXX, was a venture initiated for profits by the Blackwater founder Erik Prince.

\section{Conclusions}

In this review, we provide a synopsis of genomic structure and evolution of SARS-CoV-2, and current efforts to produce effective vaccination and therapeutic strategies for SARS-CoV-2 infection. Most therapeutic strategies are based on repurposing of existing therapeutic agents used against various virus infections and focused mainly on inhibition of the virus replication cycle, enhancement of innate immunity, and alleviation of CRS caused by COVID-19. Currently, more than 100 clinical trials on COVID-19 aim to provide robust evidence on the efficacy of the currently available anti-SARS-CoV-2 antiviral substances, such as the nucleotide analogue remdesivir, the antimalarial drug chloroquine, and drugs directed against docking of SARS-CoV-2 to the membrane-associated angiotensin converting enzyme 2 (ACE2) such as transmembrane protease serine 2 (TMPRSS2) (Table 2). The current vaccination campaign is ongoing worldwide using different types of vaccines such as Pfizer-BioNTech and Moderna, Johnson \& Johnson, Oxford-AstraZeneca, Novavax and others with an efficacy ranging from 72 to $95 \%$. In March 2021 Germany limited the use of the Oxford-AstraZeneca COVID-19 vaccine to people 60 years of age and older due to concerns that it may be causing blood clots. Further study and more data are needed to confirm the safety of different available vaccines.

Acknowledgements Figures are designed using Bio Render. 
Author contributions Wrote the manuscript: HEM; Revised the manuscript: GP, FA, CC, TC.

Funding Not applicable.

Availability of data and material Not applicable.

\section{Declarations}

Conflict of interest The authors declare that there is no conflict of interest regarding the publication of this article.

Ethical approval Not applicable.

Consent to participate Not applicable.

Consent to publication Not applicable.

\section{References}

1. Wang C, Horby PW, Hayden FG, Gao GF (2020) A novel coronavirus outbreak of global health concern. Lancet 395(10223):470-473

2. Organization WH (2005) Statement on the second meeting of the International Health Regulations (2005) Emergency Committee regarding the outbreak of novel coronavirus (2019-nCoV)

3. Mutti L, Pentimalli F, Baglio G, Maiorano P, Saladino RE, Correale $\mathrm{P}$ et al (2020) Coronavirus disease (Covid-19): what are we learning in a country with high mortality rate? Front Immunol 11:1208

4. Correale P, Mutti L, Pentimalli F, Baglio G, Saladino RE, Sileri $P$ et al (2020) HLA-B* 44 and $C^{*} 01$ prevalence correlates with Covid19 spreading across Italy. Int J Mol Sci 21(15):5205

5. Shereen MA, Khan S, Kazmi A, Bashir N, Siddique R (2020) COVID-19 infection: origin, transmission, and characteristics of human coronaviruses. J Adv Res 24:81

6. Snijder EJ, Van Der Meer Y, Zevenhoven-Dobbe J, Onderwater JJ, van der Meulen J, Koerten HK et al (2006) Ultrastructure and origin of membrane vesicles associated with the severe acute respiratory syndrome coronavirus replication complex. J Virol 80(12):5927-5940

7. Hussain S, Chen Y, Yang Y, Xu J, Peng Y, Wu Y et al (2005) Identification of novel subgenomic RNAs and noncanonical transcription initiation signals of severe acute respiratory syndrome coronavirus. J Virol 79(9):5288-5295

8. Masters PS (2006) The molecular biology of coronaviruses. Adv Virus Res 66:193-292

9. Delmas B, Laude H (1990) Assembly of coronavirus spike protein into trimers and its role in epitope expression. J Virol 64(11):5367-5375

10. Beniac DR, Andonov A, Grudeski E, Booth TF (2006) Architecture of the SARS coronavirus prefusion spike. Nat Struct Mol Biol 13(8):751-752

11. Fehr AR, Perlman S (2015) Coronaviruses: an overview of their replication and pathogenesis. Coronaviruses. Springer, Berlin, pp $1-23$

12. DeDiego ML, Álvarez E, Almazán F, Rejas MT, Lamirande E, Roberts A et al (2007) A severe acute respiratory syndrome coronavirus that lacks the $\mathrm{E}$ gene is attenuated in vitro and in vivo. $\mathrm{J}$ Virol 81(4):1701-1713
13. Nieto-Torres JL, DeDiego ML, Verdia-Baguena C, JimenezGuardeno JM, Regla-Nava JA, Fernandez-Delgado R et al (2014) Severe acute respiratory syndrome coronavirus envelope protein ion channel activity promotes virus fitness and pathogenesis. PLoS Pathog 10:5

14. Chang C-K, Sue S-C, Yu T-H, Hsieh C-M, Tsai C-K, Chiang Y-C et al (2006) Modular organization of SARS coronavirus nucleocapsid protein. J Biomed Sci 13(1):59-72

15. Hurst KR, Koetzner CA, Masters PS (2009) Identification of in vivo-interacting domains of the murine coronavirus nucleocapsid protein. J Virol 83(14):7221-7234

16. Cui L, Wang H, Ji Y, Yang J, Xu S, Huang X et al (2015) The nucleocapsid protein of coronaviruses acts as a viral suppressor of RNA silencing in mammalian cells. J Virol 89(17):9029-9043

17. Prajapat M, Sarma P, Shekhar N, Avti P, Sinha S, Kaur H et al (2020) Drug targets for corona virus: a systematic review. Indian J Pharmacol 52(1):56

18. Deng L, Li C, Zeng Q, Liu X, Li X, Zhang H et al (2020) Arbidol combined with LPV/r versus LPV/r alone against Corona Virus Disease 2019: a retrospective cohort study. J Infect 81(1):e1-e5

19. Li Q, Guan X, Wu P, Wang X, Zhou L, Tong Y et al (2020) Early transmission dynamics in Wuhan, China, of novel coronavirusinfected pneumonia. New Engl J Med 382:1199

20. Cotten M, Watson SJ, Kellam P, Al-Rabeeah AA, Makhdoom HQ, Assiri A et al (2013) Transmission and evolution of the Middle East respiratory syndrome coronavirus in Saudi Arabia: a descriptive genomic study. The Lancet 382(9909):1993-2002

21. Kannan S, Subbaram K, Ali S, Kannan H (2020) Protein in SARSCoV-1, SARS-CoV-2, MERS-CoV, and Bat Coronavirus. J Pure Appl Microbiol 14(suppl 1):757-763

22. Annan A, Baldwin HJ, Corman VM, Klose SM, Owusu M, Nkrumah EE et al (2013) Human betacoronavirus 2c EMC/2012related viruses in bats, Ghana and Europe. Emerg Infect Dis 19(3):456

23. Anthony SJ, Gilardi K, Menachery V, Goldstein T, Ssebide B, Mbabazi R et al (2017) Further evidence for bats as the evolutionary source of Middle East respiratory syndrome coronavirus. MBio 8(2):e00373-e417

24. Moreno A, Lelli D, De Sabato L, Zaccaria G, Boni A, Sozzi E et al (2017) Detection and full genome characterization of two beta $\mathrm{CoV}$ viruses related to Middle East respiratory syndrome from bats in Italy. Virol J 14(1):1-11

25. Yang L, Wu Z, Ren X, Yang F, Zhang J, He G et al (2014) MERSrelated betacoronavirus in Vespertilio superans bats, China. Emerg Infect Dis 20(7):1260

26. Market Onglyza OPCL. Merck \& Co. Inc. and Ono Pharmaceutical Co. Ltd. market Glactiv sitagliptin to treat diabetes. Novartis $\mathrm{AG}$ markets Equa vildagliptin to treat

27. Zhou G, Zhao Q (2020) Perspectives on therapeutic neutralizing antibodies against the Novel Coronavirus SARS-CoV-2. Int J Biol Sci 16(10): 1718

28. Walls AC, Park Y-J, Tortorici MA, Wall A, McGuire AT, Veesler D (2020) Structure, function, and antigenicity of the SARS-CoV-2 spike glycoprotein. Cell 181(2):281-292

29. Acter T, Uddin N, Das J, Akhter A, Choudhury TR, Kim S (2020) Evolution of severe acute respiratory syndrome coronavirus 2 (SARS-CoV-2) as coronavirus disease 2019 (COVID-19) pandemic: a global health emergency. Sci Total Environ 2020:138996

30. Wacharapluesadee S, Tan CW, Maneeorn P, Duengkae P, Zhu F, Joyjinda $\mathrm{Y}$ et al (2021) Evidence for SARS-CoV-2 related coronaviruses circulating in bats and pangolins in Southeast Asia. Nat Commun 12(1):1-9

31. Organization WH (2020) Origin of SARS-CoV-2, 26 March 2020. World Health Organization 
32. Li F (2012) Evidence for a common evolutionary origin of coronavirus spike protein receptor-binding subunits. J Virol 86(5):2856-2858

33. Hu B, Zeng L-P, Yang X-L, Ge X-Y, Zhang W, Li B et al (2017) Discovery of a rich gene pool of bat SARS-related coronaviruses provides new insights into the origin of SARS coronavirus. PLoS Pathog 13:11

34. Li F, Li W, Farzan M, Harrison SC (2005) Structure of SARS coronavirus spike receptor-binding domain complexed with receptor. Science 309(5742): 1864-1868

35. Zhang X, Li S, Niu S (2020) ACE2 and COVID-19 and the resulting ARDS. Postgrad Med J 96(1137):403-407

36. Xu X, Chen P, Wang J, Feng J, Zhou H, Li X et al (2020) Evolution of the novel coronavirus from the ongoing Wuhan outbreak and modeling of its spike protein for risk of human transmission. Sci China Life Sci 63(3):457-460

37. Schwede T, Kopp J, Guex N, Peitsch MC (2003) SWISS-MODEL: an automated protein homology-modeling server. Nucleic Acids Res 31(13):3381-3385

38. Li G, De Clercq E (2020) Therapeutic options for the 2019 novel coronavirus (2019-nCoV). Nature Publishing Group, Berlin

39. Zumla A, Chan JF, Azhar EI, Hui DS, Yuen K-Y (2016) Coronaviruses - drug discovery and therapeutic options. Nat Rev Drug Discov 15(5):327

40. Stahlmann R, Lode H (2020) Medication for COVID-19-an overview of approaches currently under study. Dtsch Arztebl Int 117(13):213

41. De Clercq E (2019) New nucleoside analogues for the treatment of hemorrhagic fever virus infections. Chemistry 14(22):3962-3968

42. Liu W, Morse JS, Lalonde T, Xu S (2020) Learning from the past: possible urgent prevention and treatment options for severe acute respiratory infections caused by $2019-\mathrm{nCoV}$. Chembiochem

43. Mulangu S, Dodd LE, Davey RT Jr, Tshiani Mbaya O, Proschan M, Mukadi D et al (2019) A randomized, controlled trial of Ebola virus disease therapeutics. N Engl J Med 381(24):2293-2303

44. Sheahan TP, Sims AC, Graham RL, Menachery VD, Gralinski LE, Case JB et al (2017) Broad-spectrum antiviral GS-5734 inhibits both epidemic and zoonotic coronaviruses. Sci Transl Med 9:396

45. Agostini ML, Andres EL, Sims AC, Graham RL, Sheahan TP, $\mathrm{Lu} \mathrm{X}$ et al (2018) Coronavirus susceptibility to the antiviral remdesivir (GS-5734) is mediated by the viral polymerase and the proofreading exoribonuclease. MBio 9(2):e00221-e318

46. Wang M, Cao R, Zhang L, Yang X, Liu J, Xu M et al (2020) Remdesivir and chloroquine effectively inhibit the recently emerged novel coronavirus (2019-nCoV) in vitro. Cell Res 30(3):269-271

47. Holshue ML, DeBolt C, Lindquist S, Lofy KH, Wiesman J, Bruce $\mathrm{H}$ et al (2020) First case of 2019 novel coronavirus in the United States. New Engl J Med 382:10

48. Wagstaff KM, Sivakumaran H, Heaton SM, Harrich D, Jans DA (2012) Ivermectin is a specific inhibitor of importin $\alpha / \beta$-mediated nuclear import able to inhibit replication of HIV-1 and dengue virus. Biochem J 443(3):851-856

49. Caly L, Druce JD, Catton MG, Jans DA, Wagstaff KM (2020) The FDA-approved Drug Ivermectin inhibits the replication of SARS-CoV-2 in vitro. Antiviral Res 2020:104787

50. Monteil V, Kwon H, Prado P, Hagelkrüys A, Wimmer RA, Stahl $M$ et al (2020) Inhibition of SARS-CoV-2 infections in engineered human tissues using clinical-grade soluble human ACE2. Cell. https://doi.org/10.1016/j.cell.2020.04.004
51. Khan A, Benthin C, Zeno B, Albertson TE, Boyd J, Christie JD et al (2017) A pilot clinical trial of recombinant human angiotensin-converting enzyme 2 in acute respiratory distress syndrome. Crit Care 21(1):234

52. Vincent MJ, Bergeron E, Benjannet S, Erickson BR, Rollin PE, Ksiazek TG et al (2005) Chloroquine is a potent inhibitor of SARS coronavirus infection and spread. Virol J 2(1):69

53. Zheng M, Gao Y, Wang G, Song G, Liu S, Sun D, Xu Y, Tian Z (2020) Functional exhaustion of antiviral lymphocytes in COVID19 patients. Cell Mol Immunol 17(5):533-535

54. Vander Heiden MG. 1, 21 Koch Institute for Integrative Cancer Research and Department of Biology, Massachusetts Institute of Technology, Cambridge, MA, USA. Department of Medical Oncology, Dana-Farber Cancer Institute, Boston, MA. USA Annual Review of Cancer Biology 4:17-40

55. Diao B, Wang C, Tan Y, Chen X, Liu Y, Ning L et al (2020) Reduction and functional exhaustion of $\mathrm{T}$ cells in patients with coronavirus disease 2019 (COVID-19). Front Immunol 11:827

56. Hotchkiss RS, Opal SM (2020) Activating immunity to fight a foe-a new path. N Engl J Med 382(13):1270-1272

57. Saag MS (2020) Misguided use of hydroxychloroquine for COVID-19: the infusion of politics into science. JAMA 324(21):2161-2162

58. Ahmed SF, Quadeer AA, McKay MR (2020) Preliminary identification of potential vaccine targets for the COVID-19 coronavirus (SARS-CoV-2) based on SARS-CoV immunological studies. Viruses 12(3): 254

59. Tu Y-F, Chien C-S, Yarmishyn AA, Lin Y-Y, Luo Y-H, Lin Y-T et al (2020) A review of SARS-CoV-2 and the ongoing clinical trials. Int J Mol Sci 21(7):2657

60. Chappell K, Watterson D, Young P (2018) Rapid response pipeline for stabilized subunit vaccines

61. Mahase E (2021) Covid-19: Novavax vaccine efficacy is $86 \%$ against UK variant and 60\% against South African variant. $\mathrm{Br}$ Med J 372:296

62. Al-Halifa S, Gauthier L, Arpin D, Bourgault S, Archambault D (2019) Nanoparticle-based vaccines against respiratory viruses. Front Immunol 10:22

63. Boytchev H (2021) Covid-19: Germany struggles with slow uptake of Oxford AstraZeneca vaccine. Br Med J 372:619

64. Logunov DY, Dolzhikova IV, Shcheblyakov DV, Tukhvatulin AI, Zubkova OV, Dzharullaeva AS et al (2021) Safety and efficacy of an rAd26 and rAd5 vector-based heterologous prime-boost COVID-19 vaccine: an interim analysis of a randomised controlled phase 3 trial in Russia. Lancet. https://doi.org/10.1016/ S0140-6736(21)00234-8

65. Gates B (2020) Responding to Covid-19-a once-in-a-century pandemic? N Engl J Med 382(18):1677-1679

66. Thomas K, LaFraniere S, Weiland N, Goodnough A, Haberman M (2020) Covid-19: FDA clears Pfizer vaccine and millions of doses will be shipped right away. The New York Times 11

Publisher's Note Springer Nature remains neutral with regard to jurisdictional claims in published maps and institutional affiliations. 